ternational Electronic Journal of Algebra

Volume 26 (2019) 191-203

DOI: $10.24330 /$ ieja. 587053

\title{
ON LEAVITT PATH ALGEBRAS OVER COMMUTATIVE RINGS
}

\author{
Pramod Kanwar, Meenu Khatkar and R. K. Sharma \\ Received: 4 March 2019; Accepted: 8 June 2019 \\ Communicated by Surender K. Jain
}

\begin{abstract}
In this article, basic ideals in a Leavitt path algebra over a commutative unital ring are studied. It is shown that for a finite acyclic graph $E$ and a commutative unital ring $R$, the Leavitt path algebra $L_{R}(E)$ is a direct sum of minimal basic ideals and that for a commutative ring $R$ and a graph $E$ satisfying Condition (L), the Leavitt path algebra $L_{R}(E)$ has no non-zero nilpotent basic ideals. Uniqueness theorems for Leavitt path algebras over commutative unital rings are also discussed.
\end{abstract}

Mathematics Subject Classification (2010): 16D70, 16G30, 16P10, 16W50

Keywords: Leavitt path algebra, basic ideal, minimal basic ideal, uniqueness theorem

\section{Introduction}

Leavitt path algebras of row-finite graphs, introduced by Abrams and Aranda Pino in [2] and independently by Ara, Moreno, and Pardo in [9], have been of interest to algebraists as well as analysts due to their connections with algebraic structures such as matrix rings, Laurent polynomial rings and also with $C^{*}$-algebras (see for example [1], [4], [5], [6], [7], [8], [10], [13], [14], [15]). Several generalizations of these algebras have also been studied in the last decade. On one hand, Abrams and Aranda Pino generalized the concept to arbitrary graphs (see [3]) and on the other, Tomforde considered these algebras where the coefficients came from a commutative unital ring in place of a field (see [16]). It is not hard to see that several results about these algebras over a field do not remain valid if we replace field with a commutative unital ring. For example, Leavitt path algebra of a finite line graph, being a matrix ring over a field, is simple; the Leavitt path algebra over a field has no non-zero nilpotent ideals; and that the Jacobson radical of a Leavitt path algebra over a field is always 0 . In the case of a commutative unital ring these results, however, are not true, in general. Tomforde showed that some of the well-known results about Leavitt path algebras over a field can be generalized to Leavitt path algebras over a commutative unital ring by suitably modifying the 
statement. To accomplish this, Tomforde introduced the concepts of basic ideals, basically simple Leavitt path algebras, among other things.

In this article, we continue the study of Leavitt path algebras over commutative unital rings and show that in the definition of basic ideals of Leavitt path algebra $L_{R}(E)$ given by Tomforde $([16])$, the vertex $v$ can be replaced by any basis element of $L_{R}(E)$ (Theorem 3.8). For a finite acyclic graph $E$, we show that Leavitt path algebra $L_{R}(E)$, is a direct sum of minimal basic ideals (Theorem 3.9) and that a graph $E$ satisfy Condition (L) if and only if every non-zero basic ideal of $L_{R}(E)$ contains a vertex (Theorem 3.13 and Theorem 3.16). It is also shown that if $R$ is a commutative unital ring and $E$ is a graph satisfying Condition (L) then the Leavitt path algebra $L_{R}(E)$ has no non-zero nilpotent basic ideals (Theorem 3.10). We also prove that for a graph having no isolated vertices and for any ring homomorphism $\phi$ from $L_{R}(E)$ to a ring, the conditions " $\phi(r v) \neq 0$ for all $v \in E^{0}$ and $0 \neq r \in R$ ", " $\phi(r e) \neq 0$ for all $e \in E^{1}$ and $0 \neq r \in R$ ", " $\phi\left(r e^{*}\right) \neq 0$ for all $e^{*} \in\left(E^{1}\right)^{*}$ and $0 \neq r \in R$ " and " $\phi(r \mu) \neq 0$ for all $\mu \in \operatorname{Path}(E)$ and $0 \neq r \in R$ " are equivalent and use these to give additional equivalent conditions in Graded Uniqueness Theorem (Theorem 4.6).

\section{Preliminaries and notation}

Throughout this article, a ring will mean a commutative unital ring and a graph will always mean a directed graph.

A graph with $E^{0}$ as the set of vertices, $E^{1}$, the set of edges, and the functions $r, s: E^{1} \rightarrow E^{0}$ is denoted by $E=\left(E^{0}, E^{1}, r, s\right)$. For each edge $e \in E^{1}$, the vertices $s(e)$ and $r(e)$ are called the source and range of $e$ respectively. For $v \in E^{0}$, a loop at $v$ is an edge $e$ for which $r(e)=s(e)$. A vertex which does not receive any edge is called a source. A vertex which does not emit any edge is called a sink. A vertex $v \in E^{0}$ such that $\left|s^{-1}(v)\right|=\infty$ is called an infinite emitter. A vertex $v$ which is either a sink or an infinite emitter is called a singular vertex. A vertex $v$ which is not a singular vertex is called a regular vertex. A vertex which is both a source and a sink is called an isolated vertex.

A path $\mu$ in a graph $E$ is a finite sequence of edges $\mu=e_{1} e_{2} \cdots e_{n}$ such that $r\left(e_{i}\right)=s\left(e_{i+1}\right)$ for $i=1,2, \ldots, n-1$. In this case, $s\left(e_{1}\right)$ is called the source of $\mu$ (denoted by $s(\mu)$ ), $r\left(e_{n}\right)$ is called the range of $\mu$ (denoted by $r(\mu)$ ), and $n$ is called the length of $\mu$. We view the elements of $E^{0}$ as paths of length 0 . An edge $e$ is an exit for a path $\mu=e_{1} e_{2} \cdots e_{n}$ if there exists $i$ such that $s(e)=s\left(e_{i}\right)$ and $e \neq e_{i}$. If $\mu=e_{1} e_{2} \ldots e_{n}$ then we denote the set $\left\{s\left(e_{i}\right), r\left(e_{i}\right): i=1,2, \ldots, n\right\}$ by $\mu^{0}$. If $\mu$ 
is a path such that $v=s(\mu)=r(\mu)$ then $\mu$ is called a closed path based at $v$. If $r(\mu)=s(\mu)$ and $s\left(e_{i}\right) \neq s\left(e_{j}\right)$ for every $i \neq j$ then $\mu$ is called a cycle. A graph without any cycles is called acyclic.

We say that a graph $E$ satisfies condition $(N E)$ if no cycle in $E$ has an exit and that it satisfies condition $(L)$ if every cycle in $E$ has an exit.

For $n \geq 2$, we denote the set of paths of length $n$ by $E^{n}$ and the set of all paths by $E^{*}$. We define a relation $\leq$ on $E^{0}$ by setting $v \leq w$ if there is a path $\mu \in E^{*}$ with $s(\mu)=v$ and $r(\mu)=w$. A subset $H$ of $E^{0}$ is called hereditary if $v \leq w$ and $v \in H$ imply $w \in H$. A hereditary set is called saturated if $s^{-1}(v) \neq \phi$ and $r\left(s^{-1}(v)\right) \subset H$ then $v \in H$.

A graph is called row-finite if every vertex emits only finite number of edges. Note that, a row-finite graph is finite if $E^{0}$ is finite.

Given a graph $E$, the extended graph of $E$ is defined as the graph $\hat{E}=\left(E^{0}, E^{1} \cup\left(E^{1}\right)^{*}, r^{\prime}, s^{\prime}\right)$ where $\left(E^{1}\right)^{*}=\left\{e_{i}^{*}: e_{i} \in E^{1}\right\}$ and $\left.r^{\prime}\right|_{E^{1}}=r, r^{\prime}\left(e_{i}^{*}\right)=$ $s\left(e_{i}\right),\left.s^{\prime}\right|_{E^{1}}=s$ and $s^{\prime}\left(e_{i}^{*}\right)=r\left(e_{i}\right)$. The elements of $\left(E^{1}\right)^{*}$ are called ghost edges.

Let $R$ be a commutative unital ring and $E$ be a graph. Following Tomforde, we define a Leavitt E-family to be the set $\left\{v, e, e^{*}: v \in E^{0}, e \in E^{1}\right\}$ in $R$ such that

(1) $v w=\delta_{v w} v$ for all $v, w \in E^{0}$,

(2) $s(e) e=e r(e)=e$ for all $e \in E^{1}$,

(3) $r(e) e^{*}=e^{*} s(e)=e^{*}$ for all $e \in E^{1}$,

(4) $e^{*} f=\delta_{e f} r(e)$ for all $e, f \in E^{1}$,

(5) $v=\sum_{e \in s^{-1}(v)} e e^{*}$ for every regular vertex $v \in E^{0}$.

Note that the Condition (2) and Condition (3) can be combined to read $s^{\prime}(e) e=$ $e r^{\prime}(e)=e$ for all $e \in E^{1} \cup\left(E^{1}\right)^{*}$. The conditions (4) and (5) are referred to as Cuntz-Kreiger relations in the literature and are denoted by (CK1) and (CK2), respectively.

The Leavitt path algebra of $E$ with coefficients in $R$, denoted by $L_{R}(E)$, is defined as the universal $R$-algebra generated by a Leavitt $E$-family.

We remark that if $E^{0}$ is finite then $L_{R}(E)$ is unital $R$-algebra with unit as sum of all the vertices. If $E^{0}$ is infinite then $L_{R}(E)$ is an algebra with local units. Also $L_{R}(E)$ is a $\mathbb{Z}$-graded algebra with grading induced by degree $\left(v_{i}\right)=0$, degree $\left(e_{i}\right)=1$, degree $\left(e_{i}{ }^{*}\right)=-1$ for all $v_{i} \in E^{0}$ and $e_{i} \in E^{1}$, that is, $L_{R}(E)=$ $\bigoplus_{n \in \mathbb{Z}} L_{R}(E)_{n}$, where $L_{R}(E)_{0}=R E^{0}+A_{0}, L_{R}(E)_{n}=A_{n}$ for $n \neq 0$ and $A_{n}=$ $\sum\left\{r e_{i_{1}} e_{i_{2}} \cdots e_{i_{\sigma}} e_{j_{1}}{ }^{*} e_{j_{2}}{ }^{*} \cdots e_{j_{\tau}}{ }^{*}: \sigma+\tau \geq 0, e_{i_{s}} \in E^{1}, e_{j_{t}}{ }^{*} \in\left(E^{1}\right)^{*}, r \in R, \sigma-\tau=\right.$ $n\}$ for all $n$. 
An element $x \in L_{R}(E)$ is called a normal element if $x x^{*}=x^{*} x$. The commutative core $M_{R}(E)$ of Leavitt path algebra $L_{R}(E)$ is defined as the commutative $R$-subalgebra of $L_{R}(E)$, generated by the normal elements of the form $\alpha \beta^{*}$, where $\alpha$ and $\beta$ are paths in $E$ having the same range.

\section{On basic ideals of Leavitt path algebras}

Let $R$ be a commutative unital ring and let $E$ be a graph. An ideal $I$ of $L_{R}(E)$ is called a basic ideal if whenever $r \in R \backslash\{0\}$ and $v \in E^{0}$, we have $r v \in I$ implies $v \in I$ (see [16]). A basic ideal of $L_{R}(E)$ is called a minimal basic ideal if it does not contain any non-zero basic ideal other than itself. The Leavitt path algebra $L_{R}(E)$ is called basically simple if the only basic ideals of $L_{R}(E)$ are $\{0\}$ and $L_{R}(E)$.

Example 3.1. If $R=\mathbb{Z}_{4}$ and $E$ is the graph
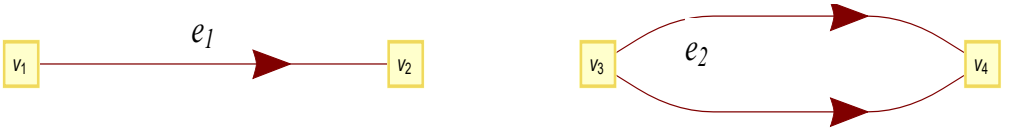

then each of the ideals $\left(\begin{array}{cc}\mathbb{Z}_{4} & \mathbb{Z}_{4} \\ \mathbb{Z}_{4} & \mathbb{Z}_{4}\end{array}\right)$ and $\left(\begin{array}{ccc}\mathbb{Z}_{4} & \mathbb{Z}_{4} & \mathbb{Z}_{4} \\ \mathbb{Z}_{4} & \mathbb{Z}_{4} & \mathbb{Z}_{4} \\ \mathbb{Z}_{4} & \mathbb{Z}_{4} & \mathbb{Z}_{4}\end{array}\right)$ is a basic ideal of the Leavitt path algebra $L_{R}(E)$ of $E$ with coefficients in $R$. We further observe that each of these is a minimal basic ideal and hence $L_{R}(E)$ is not basically simple. We also note that the ideal $\left(\begin{array}{cc}2 \mathbb{Z}_{4} & 2 \mathbb{Z}_{4} \\ 2 \mathbb{Z}_{4} & 2 \mathbb{Z}_{4}\end{array}\right)$ is not a basic ideal of $L_{R}(E)$.

We now give conditions that are equivalent to the condition $r v \in I$ implies $v \in I$ in the definition of a basic ideal. To do this we use $(v),(e)$, and $\left(e^{*}\right)$ to denote the conditions:

$(v)$ : for $r \in R \backslash\{0\}$ and $v \in E^{0}, r v \in I$ implies $v \in I$,

(e) : for $r \in R \backslash\{0\}$ and $e \in E^{1}$, re $\in I$ implies $e \in I$, and

$\left(e^{*}\right):$ for $r \in R \backslash\{0\}$ and $e^{*} \in\left(E^{1}\right)^{*}, r e^{*} \in I$ implies $e^{*} \in I$

for any ideal $I$ of $L_{R}(E)$. Note that $I$ is basic if it satisfies Condition $(v)$.

Proposition 3.2. Let $R$ be a commutative unital ring and $E$ be a graph. If I is an ideal in $L_{R}(E)$ satisfying Condition $(v)$ then I satisfies Condition $(e)$ and Condition $\left(e^{*}\right)$.

Proof. Let $I$ satisfies Condition $(v)$ and suppose $r \in R \backslash\{0\}$ and $e \in E^{1}$ be such that $r e \in I$. Then $r e^{*} e \in I$, that is, $r \cdot r(e) \in I$. Since $I$ satisfies Condition $(v)$, we get $r(e) \in I$. But then $e \cdot r(e) \in I$, that is, $e \in I$. Hence $I$ satisfies Condition $(e)$. 
Similarly, if $r e^{*} \in I$ then $r e^{*} e \in I$, that is, $r \cdot r(e) \in I$. Thus $r(e) \in I$, which is equivalent to saying that $s\left(e^{*}\right) \in I$. But then $s\left(e^{*}\right) \cdot e^{*} \in I$, that is, $e^{*} \in I$. Hence $I$ satisfies Condition $\left(e^{*}\right)$.

The converse of Proposition 3.2 is not true, in general. For example, if $R=\mathbb{Z}_{4}$ and $\mathrm{E}$ is the graph

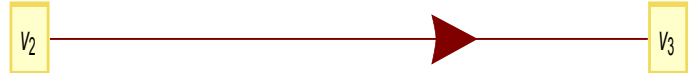

then the ideal $<2 v_{1}>$ satisfy Condition $(e)$ and Condition $\left(e^{*}\right)$, but does not satisfy Condition $(v)$. If the graph has no isolated vertices then the converse is true as shown in the following propositions (Proposition 3.3 and Proposition 3.4).

Proposition 3.3. Let $R$ be a commutative unital ring and $E$ be a graph without any isolated vertices. If $I$ is an ideal in $L_{R}(E)$ satisfying Condition $(e)$ then $I$ satisfies Condition ( $v)$.

Proof. Let $I$ satisfies Condition (e) and let $r \in R \backslash\{0\}$ and $v \in E^{0}$ be such that $r v \in I$. First assume $v$ is a sink (not necessarily a source) and let $e$ be an edge such that $r(e)=v$. Then $r v \in I$ gives $r e v \in I$. Hence $r e \in I$. Since $I$ satisfies Condition $(e)$, we get $e \in I$. Thus $e^{*} e \in I$, that is, $v \in I$. Next assume $v$ is a regular vertex and let $e$ be an edge such that $s(e)=v$. Then $r v \in I$ gives $r v e \in I$. Hence $r e \in I$. Since $I$ satisfies Condition $(e)$, we get $e \in I$. But then $e e^{*} \in I$ for every edge $e$ with $s(e)=v$. Hence $v=\sum_{\{e \mid s(e)=v\}} e e^{*} \in I$. Thus $I$ satisfies Condition $(v)$.

Proposition 3.4. Let $R$ be a commutative unital ring and $E$ be a graph without any isolated vertices. If $I$ is an ideal in $L_{R}(E)$ satisfying Condition $\left(e^{*}\right)$ then $I$ satisfies Condition $(v)$.

Proof. The proof is similar to that of Proposition 3.3.

By combining Proposition 3.2, Proposition 3.3, and Proposition 3.4, we get the following theorem.

Theorem 3.5. Let $R$ be a commutative unital ring and $E$ be a graph without any isolated vertices. Then for any ideal $I$ of $L_{R}(E)$, the following conditions are equivalent.

(1) I satisfies Condition $(v)$.

(2) I satisfies Condition (e).

(3) I satisfies Condition $\left(e^{*}\right)$. 
Corollary 3.6. Let $R$ be a commutative unital ring and $E$ be a graph without any isolated vertices. Then an ideal $I$ of $L_{R}(E)$ is basic if and only if I satisfies Condition (e) if and only if I satisfies Condition $\left(e^{*}\right)$.

Proposition 3.7. Let $R$ be a commutative unital ring, $E$ be a graph and $I$ be an ideal in $L_{R}(E)$. Then for any path $\mu$ in $L_{R}(E)$ and $r \in R \backslash\{0\}, r \mu \in I$ implies $\mu \in I$ if and only if I satisfies Condition $(v)$.

Proof. Suppose $I$ satisfies Condition $(v)$. Let $r \in R \backslash\{0\}$ and $\mu=e_{1} e_{2} \ldots e_{n}$ be a path in $L_{R}(E)$ such that $r \mu \in I$. Then $r \cdot e_{1}{ }^{*} e_{1} e_{2} \ldots e_{n} \in I$, which is equivalent to $r \cdot r\left(e_{1}\right) e_{2} \ldots e_{n} \in I$. Since $r\left(e_{1}\right)=s\left(e_{2}\right)$, we have $r \cdot e_{2} \ldots e_{n} \in I$. Again since $I$ is an ideal, $r \cdot e_{2}{ }^{*} e_{2} \ldots e_{n} \in I$. Repeating this process, finally we get $r \cdot e_{n}{ }^{*} e_{n} \in I$, that is, $r \cdot r\left(e_{n}\right) \in I$. Since $I$ satisfies Condition $(v)$, we get $r\left(e_{n}\right) \in I$ and hence $e_{n} \cdot r\left(e_{n}\right) \in I$. Thus $e_{n} \in I$. Now using the fact that $I$ is an ideal of $L_{R}(E)$, we get $e_{1} e_{2} \ldots e_{n} \in I$, that is, $\mu \in I$. Since vertices are paths of length 0 , the other direction is clear.

A similar argument can be used to prove that the result of Proposition 3.7 remains true if we replace $\mu$ with $p q^{*}$, where $p$ and $q$ are paths in $L_{R}(E)$. Since the elements of $E^{0} \cup E^{1} \cup E^{1^{*}}$ and elements of the form $p q^{*}$ where $p, q$ are paths in $L_{R}(E)$ form a basis $\mathcal{B}$ of $L_{R}(E)$ [14, Theorem 3.7], we have the following theorem.

Theorem 3.8. Let $R$ be a commutative unital ring and $E$ be a graph. Then an ideal I of $L_{R}(E)$ satisfies Condition $(v)$ if and only if for $r \in R \backslash\{0\}$ and for every basis element $x$ in $\mathcal{B}, r x \in I$ implies $x \in I$. Equivalently, $I$ is a basic ideal if and only if for $r \in R \backslash\{0\}$ and $x \in \mathcal{B}, r x \in I$ implies $x \in I$.

Recall that a subset $H$ of $E^{0}$ is called hereditary saturated if for $v, w \in E^{0}$, $v \in H$ and $v \leq w$ implies $w \in H$ and if $s^{-1}(v) \neq \phi$ for $v \in E^{0}$ and $r\left(s^{-1}(v)\right) \subset H$ then $v \in H$. Clearly, if $v$ is an isolated vertex in $E^{0}$ then $\{v\}$ is a hereditary saturated subset. Also if $v$ is a sink then $\{v\}$ is a hereditary subset of $E^{0}$ and $\operatorname{Span}_{R}\left\{\alpha \beta^{*}: r(\alpha)=r(\beta)=v\right\}$ is a basic ideal that contains only one vertex $v$. We denote this basic ideal by $I_{v}$. By [16, Lemma 7.8] there is a saturation $H$ of $\{v\}$ such that $I_{v}=I_{H}$.

We further recall that the Leavitt path algebra $L_{R}(E)$ of a graph $E$ with coefficients from a commutative unital ring $R$ is basically simple if it has no basic ideals other than $\{0\}$ and $L_{R}(E)$. For a row-finite graph $E$, Tomforde showed that $L_{R}(E)$ is basically simple if and only if $E^{0}$ has no hereditary saturated subsets other than $\phi$ and $E^{0}$ and $E$ satisfies Condition $(L)$, namely, every cycle in $E$ has 
an exit [16, Theorem 7.20]. Since a finite acyclic graph satisfies Condition $(L)$, the Leavitt path algebra $L_{R}(E)$ of a finite acyclic graph is basically simple if and only if $E^{0}$ has no hereditary saturated subsets other than $\phi$ and $E^{0}$. In general, we have the following for the Leavitt path algebras of finite acyclic graphs.

Theorem 3.9. Let $R$ be a commutative unital ring and $E$ be a finite acyclic graph. Then the Leavitt path algebra $L_{R}(E)$ is a direct sum of minimal basic ideals.

Proof. Let $v_{1}, v_{2}, \ldots, v_{n}$ be the sinks in $E$. Then $L_{R}(E) \cong \bigoplus_{i=1}^{n} I_{v_{i}}$. It is, therefore, enough to show that if $v$ is a sink then $I_{v}$ is a minimal basic ideal. So let $v$ be a sink. As observed above, there exists saturation $H$ (say) of $\{v\}$ such that $I_{v}=I_{H}$ is a graded basic ideal of $L_{R}(E)$ [16, Lemma 7.8]. To prove the minimality of the basic ideal $I_{H}$, let $J$ be a non-zero basic ideal of $L_{R}(E)$ contained in $I_{H}$. Since $I_{H}=I_{v}=\operatorname{Span}_{R}\left\{\alpha \beta^{*}: r(\alpha)=r(\beta)=v\right\}$ and $J \subset I_{H}$, it can be seen that $0 \neq r v \in J$ for some $r \in R$. Since $J$ is basic, $v \in J$. Since $J \subset I_{H}, J^{0}$, the set of vertices in $J$, is a subset of $H$. Since $v \in J^{0}$ and $J^{0}$ is a hereditary saturated subset of $E^{0}$ [16, Lemma 7.6], minimality of $H$ (being the saturation of $\{v\}$ ) gives $J^{0}=H$. But then $J=I_{H}=I_{v}$. Hence $I_{v}$ is a minimal basic ideal. It follows that $L_{R}(E)$ is the direct sum of minimal basic ideals.

It is known that the Leavitt path algebra $L_{K}(E)$ over a field $K$ has no non-zero nilpotent ideals (see [3, Proposition 6.1]). More generally, if $R$ is a commutative unital ring with no non-zero nilpotent elements (equivalently, if $R$ is a commutative semiprime unital ring) then the Leavitt path algebra $L_{R}(E)$ over $R$ has no nonzero nilpotent left, right, or two-sided nilpotent ideals (see [12, Proposition 4.5]). In general, this result is not true for Leavitt path algebras over arbitrary commutative unital rings. For example, if $R=\mathbb{Z}_{4}$ and $E$ is the graph

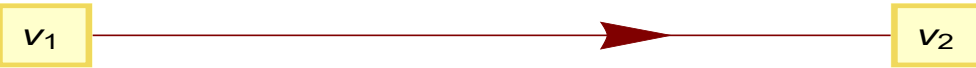

then $\left(\begin{array}{cc}2 \mathbb{Z}_{4} & 2 \mathbb{Z}_{4} \\ 2 \mathbb{Z}_{4} & 2 \mathbb{Z}_{4}\end{array}\right)$ is a non-zero nilpotent ideal of $L_{R}(E)$. We, however, have the following theorem.

Theorem 3.10. Let $R$ be a commutative unital ring and $E$ be a graph satisfying Condition $(L)$. Then the Leavitt path algebra $L_{R}(E)$ has no non-zero nilpotent basic ideals.

Proof. Let $I$ be a non-zero basic ideal of $L_{R}(E)$ and let $I^{2}=\{0\}$. By [16, Lemma 7.6], the set $I^{0}$ of vertices in $I$ is a hereditary saturated subset. By [16, Lemma 7.9], 
the ideal $<I^{0}>$ generated by $I^{0}$ is a graded basic ideal. Since $I^{0} \subset I,<I^{0}>\subset I$. But $I^{2}=\{0\}$. Thus $<I^{0}>^{2}=\{0\}$. Since $<I^{0}>$, being a graded basic ideal, is idempotent, $<I^{0}>=\{0\}$. Thus $I^{0}=\phi$. Hence $I$ does not contain any vertex, a contradiction by Proposition 3.13. Hence $I$ is not nilpotent.

We recall that $L_{R}(E)$ is $\mathbb{Z}$-graded with grading induced by degree $(v)=0$ for any vertex $v$ in $E^{0}$ and degree $(e)=1$, degree $\left(e^{*}\right)=-1$ for every $e \in E^{1}$. If $\alpha$ is a nonzero homogeneous element in $L_{R}(E)$ then there exits a path $\beta$ such that $0 \neq \alpha \beta$ is homogeneous and is in real edges only (see Theorem 2.2.11 and Corollary 2.2.12 in [1] and Theorem 5.1 in [11]). Thus $\alpha \beta=\sum_{i=1}^{n} r_{i} \gamma_{i}$, where $0 \neq r_{i} \in R$ and $\gamma_{i}$ 's are distinct paths of equal length. Since $\gamma_{1}^{*} \gamma_{1}=r\left(\gamma_{1}\right)$ and $\gamma_{1}^{*} \gamma_{i}=0$ for $2 \leq i \leq n$, it follows that $\gamma_{1}^{*} \alpha \beta=r_{1} \cdot r\left(\gamma_{1}\right)$. We, thus, have the following proposition.

Proposition 3.11. Let $R$ be a commutative unital ring and $E$ be an arbitrary graph. Let $\alpha$ be a non-zero homogeneous element of $L_{R}(E)$. Then there exist $\beta, \gamma \in \operatorname{Path}(E), 0 \neq r \in R$, and $v \in E^{0}$ such that $0 \neq \gamma^{*} \alpha \beta=r v$.

Since for a basic ideal $r v \in I$ implies $v \in I$, we have the following corollary.

Corollary 3.12. Let $R$ be a commutative unital ring and $E$ be an arbitrary graph. Every non-zero graded basic ideal of $L_{R}(E)$ contains a vertex.

In the case of graphs satisfying Condition (L), every non-zero basic ideal contains a vertex as is shown in the following theorem.

Theorem 3.13. Let $R$ be a commutative unital ring and $E$ be an arbitrary graph satisfying Condition $(L)$. Then every non-zero basic ideal of $L_{R}(E)$ contains a vertex.

Proof. Let $I$ be a non-zero basic ideal of $L_{R}(E)$ and let $\alpha$ be a non-zero element in $I$. Since $E$ satisfies Condition (L), $E$ has no cycles without exits and hence, by [11, Theorem 5.1], there exists $\mu, \eta \in \operatorname{Path}(E)$ such that either $0 \neq \mu^{*} \alpha \eta=r v$ for some $0 \neq r \in R$, and $v \in E^{0}$. Since $I$ is a basic ideal and $0 \neq r v=\mu^{*} \alpha \eta \in I$, we have $v \in I$. Thus $I$ contains a vertex.

Before we prove the converse of the Proposition 3.13 we prove a lemma which is also of independent interest. The proof of lemma is similar to that of Lemma 2.7.1 in [1]. We include it here for the sake of completeness.

Lemma 3.14. Let $R$ be a commutative unital ring and $E$ be a graph. Let $c$ be a cycle without exits such that $s(c)=v$ and $\wedge_{v}$ be a set of paths in $E$ which ends 
in $v$ but which do not contain all edges of $c$. If $I$ is a basic ideal generated by the vertices of $c$ then $I \cong M_{\wedge_{v}}\left(R\left[x, x^{-1}\right]\right)$.

Proof. Let $J$ be the basic ideal generated by $v$. Clearly $J \subseteq I$. Now for any $w \in c^{0}$ if $\mu$ denotes the portion of cycle $c$ from $v$ to $w$ then $\mu$ is a path from $v$ to $w$ and $w=\mu^{*} v \mu$. Thus $w \in J$. Hence $J$ contains all vertices of $c$. Thus $I \subseteq J$ and hence $I=J$. Now let $A$ be the set of elements of the form $\alpha c^{k} \beta^{*}$ where $\alpha, \beta \in \wedge_{v}$ and $k \in \mathbb{Z}$ where $c^{k}=v$ for $k=0$ and $c^{k}=\left(c^{*}\right)^{-k}$ for $k<0$. Since the elements of the form $\alpha \beta^{*}$ where $\alpha, \beta$ are paths form a $R$-basis of $L_{R}(E)$ [14, Theorem 3.7], $A$ is a $R$-linearly independent set. Also every element of $J$ is a $R$ linear combination of elements of the form $p q^{*}$, where $p, q$ are paths in $E$ such that $r(p)=r(q) \in T(v)$ (the tree of $v$ ). Since $c$ has no exits, $T(v)$ is precisely the set of vertices in $c$. Hence $p=\alpha c^{l}$ and $q=\beta c^{m}$ for some $\alpha, \beta \in \wedge_{v}$ and $l, m \geq 0$. Thus $A$ generates the basic ideal $J$. Now let $\phi: J \rightarrow M_{\wedge_{v}}\left(R\left[x, x^{-1}\right]\right)$ is the map defined as $\phi\left(\alpha c^{k} \beta^{*}\right)=x^{k} e_{\alpha, \beta}$ where $e_{\alpha, \beta}$ denotes the element of $M_{\wedge_{v}}\left(R\left[x, x^{-1}\right]\right)$ which is 1 in the $(\alpha, \beta)$ entry and zeros otherwise. Clearly $\phi$ is an $R$-algebra isomorphism. Hence $I=J \cong M_{\wedge_{v}}\left(R\left[x, x^{-1}\right]\right)$.

We also observe that if $I$ is a graded basic ideal of $L_{R}(E)$ then by [16, Theorem 7.9], there exists a hereditary saturated subset $H$ in $E^{0}$ such that $I=I_{H}$. But then by [16, Theorem 7.15], $I$ has a set of local units. By [16, Lemma 4.14], every ideal of $I$ is also an ideal of $L_{R}(E)$. We, thus, have the following proposition.

Proposition 3.15. Let $R$ be a commutative unital ring and $E$ be a graph. Every ideal of a graded basic ideal of the Leavitt path algebra $L_{R}(E)$ is also an ideal of $L_{R}(E)$.

Theorem 3.16. Let $R$ be a commutative unital ring and $E$ be an arbitrary graph. If every non-zero basic ideal of $L_{R}(E)$ contains a vertex then $E$ satisfies condition (L).

Proof. On the contrary, let $c$ be a cycle without exits in $E$ and let $I$ be the graded basic ideal of $L_{R}(E)$ generated by the vertices of $c$. By Lemma 3.14, there exists some vertex $v$ in $c$ such that $I \cong M_{\wedge}\left(R\left[x, x^{-1}\right]\right)$ for some set $\wedge$ of paths in $E$ which ends in $v$ but which do not contain all edges of $c$. Also by Proposition 3.15, the ideals of $I$ are ideals of $L_{R}(E)$. Since every non-zero basic ideal of $L_{R}(E)$ contains a vertex, every non-zero basic ideal of $M_{\wedge}\left(R\left[x, x^{-1}\right]\right)$ contains a non-zero idempotent, a contradiction. Hence $E$ satisfies condition (L). 


\section{Uniqueness theorems}

We recall that for a Leavitt path algebra $L_{K}(E)$ of a graph $E$, a graded homomorphism $\phi: L_{K}(E) \rightarrow A$ is injective if $\phi(v) \neq 0$ for all $v \in E^{0}$ (Graded Uniqueness Theorem). In the case of Leavitt path algebras over rings, a graded homomorphism $\phi: L_{R}(E) \rightarrow A$ is injective if $\phi(r v) \neq 0$ for all $v \in E^{0}$ and $0 \neq r \in R$ [16, Theorem 5.3]. We show that the condition $\phi(r v) \neq 0$ for all $v \in E^{0}$ and $0 \neq r \in R$ is equivalent to $\phi(r e) \neq 0$ for all $e \in E^{1}$ and $0 \neq r \in R$ as well as $\phi\left(r e^{*}\right) \neq 0$ for all $e^{*} \in E^{1^{*}}$ and $0 \neq r \in R$ in case the graph $E$ has no isolated vertices. First we prove the following proposition.

Proposition 4.1. Let $R$ be a commutative unital ring, $S$ be any ring, and $E$ be a graph. Let $\phi: L_{R}(E) \rightarrow S$ be a ring homomorphism such that $\phi(r v) \neq 0$ for all $v \in E^{0}$ and $0 \neq r \in R$. Then $\phi(r e) \neq 0$ for all $e \in E^{1}$ and $0 \neq r \in R$ and $\phi\left(r e^{*}\right) \neq 0$ for all $e^{*} \in\left(E^{1}\right)^{*}$ and $0 \neq r \in R$.

Proof. We first prove that $\phi(r e) \neq 0$ for all $e \in E^{1}$ and $0 \neq r \in R$. If there exists $e \in E^{1}$ and $0 \neq r \in R$ such that $\phi(r e)=0$ then $\phi\left(r e^{*} e\right)=\phi\left(e^{*}\right) \phi(r e)=0$. Since $e^{*} e=r(e)$, we have $\phi(r \cdot r(e))=0$, a contradiction, as $\phi(r v) \neq 0$ for all $v \in E^{0}$ and $0 \neq r \in R$. Hence $\phi(r e) \neq 0$ for all $e \in E^{1}$ and $0 \neq r \in R$. The other part can be proved similarly.

Proposition 4.2. Let $R$ be a commutative unital ring, $S$ be any ring, and $E$ be a graph. Let $\phi: L_{R}(E) \rightarrow S$ be a ring homomorphism such that $\phi(r v) \neq 0$ for all $v \in E^{0}$ and $0 \neq r \in R$. Then $\phi(r \mu) \neq 0$ for all $\mu \in \operatorname{Path}(E)$ and $0 \neq r \in R$.

Proof. Let, if possible, there is a path $\mu=e_{1} e_{2} \ldots e_{n}$ in $L_{R}(E)$ and $0 \neq r \in R$ such that $\phi(r \mu)=0$, that is, $\phi\left(r \cdot e_{1} e_{2} \ldots e_{n}\right)=0$. Then $\phi\left(e_{n}{ }^{*} e_{n-1}^{*} \ldots e_{1}{ }^{*}\right) \phi\left(r e_{1} e_{2} \ldots e_{n}\right)=$ 0 , which is equivalent to $\phi\left(r e_{n}{ }^{*} e_{n-1}^{*} \ldots e_{1}{ }^{*} e_{1} e_{2} \ldots e_{n}\right)=0$. Since $r\left(e_{1}\right)=s\left(e_{2}\right)$, we have $\phi\left(r e_{n}{ }^{*} e_{n-1}^{*} \ldots e_{2}{ }^{*} r\left(e_{1}\right) e_{2} e_{3} \ldots e_{n}\right)=0$. Repeating this process, finally we get $\phi\left(r e_{n}{ }^{*} e_{n}\right)=0$, that is, $\phi\left(r \cdot r\left(e_{n}\right)\right)=0$, a contradiction, as $\phi(r v) \neq 0$ for all $v \in E^{0}$ and $0 \neq r \in R$. Hence $\phi(r \mu) \neq 0$ for all $\mu \in \operatorname{Path}(E)$ and $0 \neq r \in R$.

Proposition 4.3. Let $R$ be a commutative unital ring, $S$ be any ring, and $E$ be a graph without any isolated vertices. Let $\phi: L_{R}(E) \rightarrow S$ be a ring homomorphism such that $\phi(r e) \neq 0$ for all $e \in E^{1}$ and $0 \neq r \in R$. Then $\phi(r v) \neq 0$ for all $v \in E^{0}$ and $0 \neq r \in R$.

Proof. Let, if possible, there exists $v \in E^{0}$ and $0 \neq r \in R$ such that $\phi(r v)=0$.

If $v$ is a sink then since $v$ is not an isolated vertex it must be the range of some 
edge ( say $f$ ). Thus $\phi(r f)=\phi(r f v)=\phi(f) \phi(r v)=0$, a contradiction, as $\phi(r e) \neq 0$ for all $e \in E^{1}$ and $0 \neq r \in R$. Hence $\phi(r v) \neq 0$ for all $v \in E^{0}$ and $0 \neq r \in R$. If $v$ is a regular vertex then $s^{-1}(v)$ has at least one edge. Let $e \in s^{-1}(v)$. Then $\phi(r e)=\phi(r v e)=\phi(r v) \phi(e)=0$, a contradiction again. Hence $\phi(r v) \neq 0$ for all $v \in E^{0}$ and $0 \neq r \in R$.

A similar argument can be used to prove the following proposition.

Proposition 4.4. Let $R$ be a commutative unital ring, $S$ be any ring, and $E$ be a graph without any isolated vertices. Let $\phi: L_{R}(E) \rightarrow S$ be a ring homomorphism such that $\phi\left(r e^{*}\right) \neq 0$ for all $e^{*} \in\left(E^{1}\right)^{*}$ and $0 \neq r \in R$. Then $\phi(r v) \neq 0$ for all $v \in E^{0}$ and $0 \neq r \in R$.

Combining Proposition 4.1, Proposition 4.2, Proposition 4.3, and Proposition 4.4 , we have the following theorem.

Theorem 4.5. Let $R$ be a commutative unital ring, $S$ be any ring, and $E$ be a graph without any isolated vertices. Let $\phi: L_{R}(E) \rightarrow S$ be a ring homomorphism. Then the following conditions are equivalent.

(1) $\phi(r v) \neq 0$ for all $v \in E^{0}$ and $0 \neq r \in R$.

(2) $\phi(r e) \neq 0$ for all $e \in E^{1}$ and $0 \neq r \in R$.

(3) $\phi\left(r e^{*}\right) \neq 0$ for all $e^{*} \in\left(E^{1}\right)^{*}$ and $0 \neq r \in R$.

(4) $\phi(r \mu) \neq 0$ for all $\mu \in \operatorname{Path}(E)$ and $0 \neq r \in R$.

Recall that for a graph $E, M_{R}(E)$ denotes the commutative $R$-subalgebra of $L_{R}(E)$, generated by the normal elements of the form $\alpha \beta^{*}$ where $\alpha, \beta$ are paths in $E$ with the same range. Using Theorem 4.5 and [11, Theorem 5.4], Graded Uniqueness Theorem takes the following form.

Theorem 4.6. Let $R$ be a commutative unital ring, $S$ be a graded ring, and $E$ be a graph without any isolated vertices. Let $\phi: L_{R}(E) \rightarrow S$ be a graded homomorphism. Then the following conditions are equivalent.

(1) $\phi$ is injective.

(2) Restriction of $\phi$ over the commutative core $M_{R}(E)$ is injective.

(3) $\phi(r v) \neq 0$ for all $v \in E^{0}$ and $0 \neq r \in R$.

(4) $\phi(r e) \neq 0$ for all $e \in E^{1}$ and $0 \neq r \in R$.

(5) $\phi\left(r e^{*}\right) \neq 0$ for all $e^{*} \in\left(E^{1}\right)^{*}$ and $0 \neq r \in R$.

(6) $\phi(r \mu) \neq 0$ for all $\mu \in \operatorname{Path}(E)$ and $0 \neq r \in R$.

For graphs without isolated vertices and satisfying Condition (L), we have the following theorem. 
Theorem 4.7. Let $R$ be a commutative unital ring, $S$ be any ring, and $E$ be a graph without any isolated vertices such that $E$ satisfies Condition $(L)$. Let $\phi: L_{R}(E) \rightarrow$ $S$ be a ring homomorphism. Then the following conditions are equivalent.

(1) $\phi$ is injective.

(2) Restriction of $\phi$ over the commutative core $M_{R}(E)$ is injective.

(3) $\phi(r v) \neq 0$ for all $v \in E^{0}$ and $0 \neq r \in R$.

(4) $\phi(r e) \neq 0$ for all $e \in E^{1}$ and $0 \neq r \in R$.

(5) $\phi\left(r e^{*}\right) \neq 0$ for all $e^{*} \in\left(E^{1}\right)^{*}$ and $0 \neq r \in R$.

(6) $\phi(r \mu) \neq 0$ for all $\mu \in \operatorname{Path}(E)$ and $0 \neq r \in R$.

Proof. Follows by Theorem 4.5 and [11, Corollary 5.3].

\section{References}

[1] G. Abrams, P. Ara and M. S. Molina, Leavitt Path Algebras, Lecture Notes in Mathematics, 2191, Springer, London, 2017.

[2] G. Abrams and G. Aranda Pino, The Leavitt path algebra of a graph, J. Algebra, 293(2) (2005), 319-334.

[3] G. Abrams and G. Aranda Pino, The Leavitt path algebras of arbitrary graphs, Houston J. Math., 34(2) (2008), 423-442.

[4] G. Abrams, G. Aranda Pino and M. S. Molina, Finite dimensional Leavitt path algebras, J. Pure Appl. Algebra, 209(3) (2007), 753-762.

[5] G. Abrams and Z. Mesyan, Simple Lie algebras arising from Leavitt path algebra, J. Pure Appl. Algebra, 216(10) (2012), 2302-2313.

[6] A. Alahmedi and H. Alsulami, On the simplicity of the Lie algebra of a Leavitt path algebra, Comm. Algebra, 44(9) (2016), 4114-4120.

[7] A. Alahmedi, H. Alsulami, S. K. Jain and E. Zelmanov, Leavitt path algebras of finite Gelfand-Kirillov dimension, J. Algebra Appl., 11(6) (2012), 1250225 (6 pp).

[8] A. Alahmedi, H. Alsulami, S. K. Jain and E. Zelmanov, Structure of Leavitt path algebras of polynomial growth, Proc. Natl. Acad. Sci. USA, 110(38) (2013), 15222-15224.

[9] P. Ara, M. A. Moreno and E. Pardo, Nonstable K-theory for graph algebras, Algebr. Represent. Theory, 10(2) (2007), 157-178.

[10] G. Aranda Pino and K. Crow, The center of a Leavitt path algebra, Rev. Mat. Iberoam., 27(2) (2011), 621-644.

[11] C. Gil Canto and A. Nasr-Isfahani, The maximal commutative subalgebra of a Leavitt path algebra, arXiv:1510.03992v1 [math.RA]. 
[12] P. Kanwar, M. Khatkar and R. K. Sharma, Basic one sided ideals of Leavitt path algebras over commutative rings, preprint, submitted.

[13] H. Larki, Ideal structure of Leavitt path algebras with coefficients in a unital commutative ring, Comm. Algebra, 43(12) (2015), 5031-5058.

[14] V. Lopatkin and T. G. Nam, On the homological dimensions of Leavitt path algebras with coefficients in commutative rings, J. Algebra, 481 (2017), 273292.

[15] Z. Mesyan, Commutator Leavitt path algebras, Algebr. Represent. Theory, 16(5) (2013), 1207-1232.

[16] M. Tomforde, Leavitt path algebras with coefficients in a commutative ring, J. Pure Appl. Algebra, 215(4) (2011), 471-484.

Pramod Kanwar (Corresponding Author)

Department of Mathematics

Ohio University-Zanesville

Zanesville, Ohio, USA

e-mail: kanwar@ohio.edu

Meenu Khatkar and R. K. Sharma

Department of Mathematics

Indian Institute of Technology Delhi

New Delhi, 110016, India

e-mails: meenukhatkar@gmail.com (M. Khatkar)

rksharmaiitd@gmail.com (R. K. Sharma) 